\title{
The Effectiveness of Mindfulness Therapy by VR (Virtual-Reality) With a Focus on Death Anxiety in a Patient With Cerebellar Cancer (Case Report)
}

\author{
Shahin Fahiminia ${ }^{1}$, Afshin Salahiyan ${ }^{2 *}$, Mahtab Norouzi ${ }^{1}$ \\ ${ }^{1}$ Islamic Azad University of Science and Research, Cancer Institute of Imam Khomeini Hospital, Tehran, Iran \\ ${ }^{2}$ Department of Psychology, Payame Noor University (PNU), Tehran, Iran
}

\begin{abstract}
Background: Cancer has many potential physical, psychological and social consequences for the patient. This single case study explored the efficiency of mindfulness therapy with the aid of virtual reality (VR) focusing on minimizing anxiety especially death anxiety in a patient with cerebellar cancer.

Methods: In this case study research the patient met and consulted 3 times in the first week, and the aim of these meetings was to break the patient's resistance through meditation and psychological treatment. After that, 8 sessions each one 2 hours were planned at the patient's home. In the second week, a subsidiary device called VR (large eye crystal capable of playing 3D film) was used as a method to assist the patient.

Results: The data collected from the pre/posttest of Beck anxiety showed significant progress in blood pressure, heart rate and body temperature, and increased up to its normal levels. In addition, the sense of satisfaction expressed by the patient during the sessions was noticeably significant.

Conclusion: According to results mindfulness therapy by VR with a focus on anxiety reduction as a complementary therapy has remarkable efficacy on improving quality of life in the cancer patient. Keywords: Cerebellum cancer; Death anxiety; Miindfulness; Virtual-reality (VR).
\end{abstract}

*Correspondence to Afshin Salahiyan, Assistant professor, Department of Psychology, Payame Noor University (PNU), Tehran, Iran. Tel: +989134023426 Email: salahyanafshin@yahoo. com

Published online 20 March 2019

Citation: Fahiminia S, Salahiyan A, Norouzi M. The effectiveness of mindfulness therapy by VR (virtual-reality) with a focus on death anxiety in a patient with cerebellar cancer (case report). Int Clin Neurosci J. 2019;6(1):33-35. doi: 10.15171/icnj.2019.07.

\section{Introduction}

Cancer can be the causes of the major psychological challenge for individuals. A meta-analysis of psychiatric disorder in oncological and hematological settings yielded a prevalence of psychiatric disorder of $30 \%$ to $40 \%{ }^{1}$ In the coming decades, a great increase is expected in the number of people suffering from cancer. ${ }^{2}$ Cancer has many potential physical, psychological and social consequences for the patient. Considering all three dimensions can make a different quality of life for cancer patients. Death anxiety is one of the most important variables that affects the quality of patient's life. since death anxiety is a psychological component, psychological therapies can manage death anxiety in affected patients. The potential effects of cancer screening from the psychological point of view, such as anxiety and distress have contributed to revised screening recommendations for some type of cancers, including breast and colon cancer $^{3,4}$ penitents suffering from cancer have many source of afflictions One of these is an unspecifiable fear about death, which is an existential issue. ${ }^{5}$ Gonen et $a l^{6}$ have investigated the relationship between death anxiety and its correlates in cancer patients. Death anxiety was associated with anxiety, depressive symptoms, and beliefs about what will happen after death. This study shows death anxiety could not be regarded as a natural consequence of having cancer; it is associated with the unresolved psychological and physical distress. About gender differences, studies show there is no significant relationship between death anxiety among male and female cancer patients. Patients with increased death anxieties should be given psychological interventions along with proper medical care to increase their chances of survival. ${ }^{6}$ So many studies accentuate that psychological interventions and medical cares should concurrent among each other. There are many approaches in psychology which can help cancer patients during their treatment, one of them is mindfulness and There are generally three main components of mindfulness intention, attention, and attitude. ${ }^{7}$ Results show promise for mindfulness-based interventions to treat common

(C) 2019 The Author(s). This is an open access article distributed under the terms of the Creative Commons Attribution License (http:// creativecommons.org/licenses/by/4.0/), which permits unrestricted use, distribution, and reproduction in any medium, provided the original work is properly cited. 
psychological problems such as anxiety, stress, and depression in cancer survivors and to improve overall quality of life. ${ }^{8}$

In the domain of health, the virtual reality (VR) method covers a wide range of applications from diagnosis to treatment, counseling, rehabilitation, and hospital design. In addition to the apparent manifestations, death anxiety decreases and overall anxiety that the patient experiences due to his or her living conditions is also reduced. VR is a method to gain awareness about the factors that worsen or improve the symptoms of the disease. Evaluation and study of various psychological problems, including anxiety disorders, are the result of treatment based on VR.

Bailey and Cushing postulated that, "the histogenesis of the brain furnishes the indispensable background for an understanding of its tumors" . The cerebellar cortex is a remarkably simple laminar structure ${ }^{10}$ The most prevalent neuronal subclass in the cerebellum, indeed within the entire mammalian central nervous system, is the cerebellar granule neuron. Granule neurons serve an essential role in coordinating afferent input to, and motor output from, the cerebellum through their excitatory connections with Purkinje neurons. While the role(s) of the cerebellum in sensorimotor functions, balance control and the vestibular ocular reflex have long been appreciated, recent studies have revealed a role for the cerebellum in a wide range of cognitive functions, including feed-forward sensory-motor learning, speech and spatial memory. ${ }^{11-16}$ Therefore, cerebellar cancer can cause a wide range of disabilities, which also affects patients in various physical, social, and psychological dimensions.

\section{Methods}

This single case study explored the efficiency of mindfulness therapy with the aid of VR (head-set) focusing on minimizing anxiety especially death anxiety in a patient with cerebellar cancer. The patient was a 50-year-old married woman who takes about a year to begin the symptoms of cerebellar erosion, due to the presence of a cancerous tumor. In the beginning the only thing had caused her problem was complicated movements but science this illness has the dementia property her ability in simple movements missed during the 6 months. Even speaking and any voluntary movements get her into trouble. Initially denial of disease prevented her from treatment but as the progression of disease accelerated death anxiety forced her to accept the treatment. For psychological interventions, the patient met and consulted three times in the first week, and the aim of these meetings was to break the patient's resistance through meditation and psychological treatment. After that, 8 sessions each one 2 hours were planned at the patient's home that would not interfere with the hours of medical treatment, grooming, nourishment and sleep. Every day one hour assigned to create the rapport between therapist and client, based on mindfulness methods. In the second week, a subsidiary device called VR (large eye crystal capable of playing 3D film) was used as a method to assist the patient.

The patient who was unable to walk, talk, and have a normal life, with the help of the program, she felt was attending a sporting event and was able to climb, swim, etc. She experienced that, for twice a week about 15 to 20 minutes. After that, the heart rate, blood pressure and body temperature, as well as the Beck Anxiety Inventory, which were measured before and after the mental awareness sessions were compared.

\section{Results}

The data collected from the pre-test-post-test of Beck anxiety showed significant progress in blood pressure, heart rate and body temperature, and increased up to its normal levels (Figure 1). In addition, the sense of satisfaction expressed by the patient during the sessions was noticeably significant. According to the observations, the patient was sweating a little after training because of

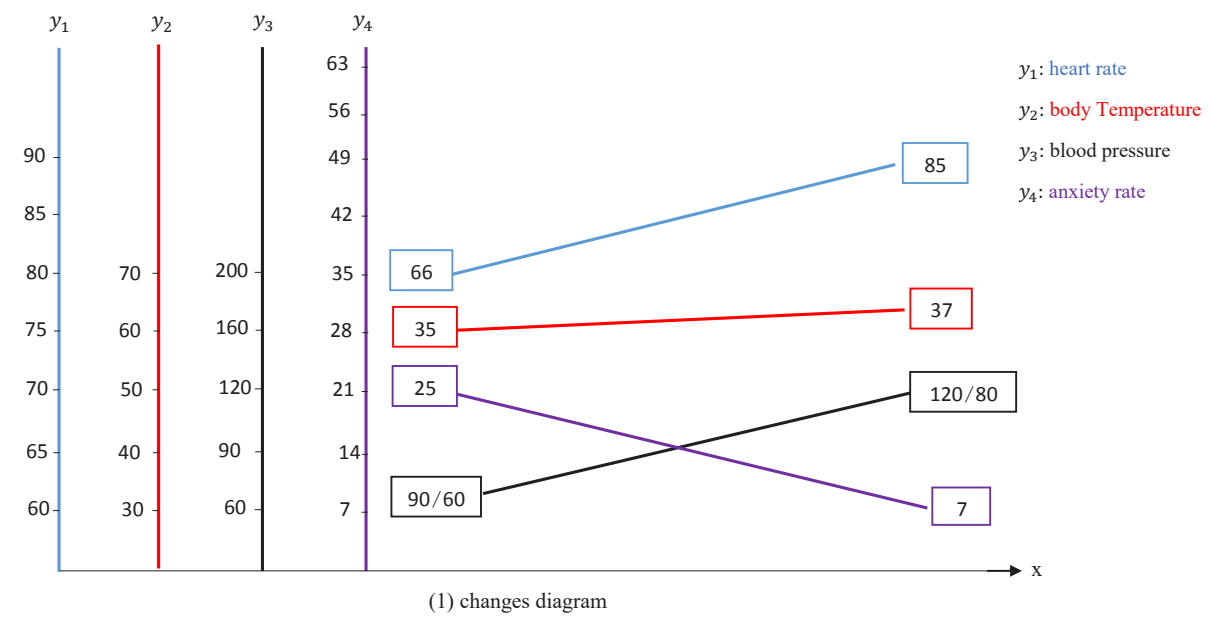

Figure 1. Changes Diagram. 
excitement which experienced during the training. The satisfaction of the client and her excitement to continue the therapy show the effectiveness of the treatment and the return of life expectancy to the patient despite difficult physical conditions. The positive result of this treatment is primarily effective on the client, and secondarily, the relatives of the patient found a way to help their patients, to have recreation without having to transfer her from the bed. This research would be a way to conformity patients with technology and using the virtual reality world tool to fill their emotional emptiness during the hospitalization.

\section{Discussion}

Alfred Adler says, "Every person has a unique personality who implemented this uniqueness". In fact, the person is both illustration and illustrator, and if a person become able to change his self-image then will become able to change the illustration which is imagining.

In this research, patient was depicted as losers who could not afford even simple duties before treatment but During the treatment, she was doing things that they did not go through in normal life. Regarding to the observable physical changes recorded, and Beck anxiety tests, which were re-started before treatment and at the end of the eighth session, showed a significant difference in the reduction of anxiety.

Mindfulness through virtual reality with an emphasis on reducing anxiety (death anxiety) as a complementary treatment to improve the life of a patient with cerebellar cancer has a significant effect.

\section{Limitations}

The present study has several limitations that must be considered along with these results. Although the observed and measured physical changes that were recorded and the pre-test and post-test Beck anxiety show the efficacy of the treatment is significant, sometimes we faced depressive mood during the treatment and its reason was the chronic and dementia property of its illness so at that situations we tried to return the mood by mindfulness methods.

Nowadays, virtual reality has been considered as a useful method in all aspects of medical, educational, diagnostic and therapeutic. However, the existing methods are in the early stages and it is necessary to use experimental methods before we widely use it. Another factor mentioned in the article is the cost of this therapeutic approach (VR-device) which we have to overcoming this obstacle before coming inclusively in clinical use.

\section{Conflict of Interest Disclosures}

The authors declare that they have no conflict of interests.

\section{Ethical Statement}

The present study was approved by Islamic Azad University of Science and Research. Additionally, the patient participated with informed consent, and she would have been able to refusal at each stage of the research.

\section{References}

1. Mitchell AJ, Chan M, Bhatti H, Halton M, Grassi L, Johansen $\mathrm{C}$, et al. Prevalence of depression, anxiety, and adjustment disorder in oncological, haematological, and palliativecare settings: a meta-analysis of 94 interview-based studies. Lancet Oncol. 2011;12(2):160-74. doi: 10.1016/s14702045(11)70002-x.

2. Bray F, Soerjomataram I. The Changing Global Burden of Cancer: Transitions in Human Development and Implications for Cancer Prevention and Control. In: Gelband H, Jha P, Sankaranarayanan R, Horton S, eds. Cancer: Disease Control Priorities, Third Edition (Volume 3). Washington (DC): The International Bank for Reconstruction and Development / The World Bank; 2015.

3. Chad-Friedman E, Coleman S, Traeger LN, Pirl WF, Goldman R, Atlas SJ, et al. Psychological distress associated with cancer screening: A systematic review. Cancer. 2017;123(20):388294. doi: 10.1002/cncr.30904.

4. Brennan M, Houssami N. Discussing the benefits and harms of screening mammography. Maturitas. 2016;92:150-3. doi: 10.1016/j.maturitas.2016.08.003.

5. Gonen G, Kaymak SU, Cankurtaran ES, Karslioglu EH, Ozalp $\mathrm{E}$, Soygur $\mathrm{H}$. The factors contributing to death anxiety in cancer patients. J Psychosoc Oncol. 2012;30(3):347-58. doi: 10.1080/07347332.2012.664260.

6. Chacko N, Sunny J. Gender Difference in Death Anxiety among Cancer Patients. Int J Indian Psychol. 2018;6(2):77-82. doi: 10.25215/0602.050.

7. Shapiro SL, Carlson LE. The Art and Science of Mindfulness: Integrating Mindfulness into Psychology and the Helping Professions. Washington, DC; American Psychological Association Publications; 2009.

8. Carlson LE. Mindfulness in Cancer Care: Hype or Help? The ASCO Post. July 10, 2018.. http://www.ascopost.com/issues/ july-10-2018/mindfulness-in-cancer-care/.

9. A classification of the tumours of the glioma group on a histogenetic basis, with a correlated study of prognosis. By Percival Bailey and Harvey Cushing. Medium 8vo. Pp. 175, with 108 illustrations. 1926. Philadelphia, London, and Montreal: J. B. Lippincott Company. 21s. net. BJS. 1927;14(55):554-5. doi: doi:10.1002/bjs.1800145540.

10. Palay SL, Chan-Palay V. Cerebellar Cortex: Cytology and Organization. Springer-Verlag; 1974.

11. Boyden ES, Katoh A, Raymond JL. Cerebellum-dependent learning: the role of multiple plasticity mechanisms. Annu Rev Neurosci. 2004;27:581-609. doi: 10.1146/annurev. neuro.27.070203.144238.

12. Hatten ME, Roussel MF. Development and cancer of the cerebellum. Trends Neurosci. 2011;34(3):134-42. doi: 10.1016/j.tins.2011.01.002.

13. Fiez JA, Petersen SE. Neuroimaging studies of word reading. Proc Natl Acad Sci U S A. 1998;95(3):914-21.

14. Schmahmann JD, Caplan D. Cognition, emotion and the cerebellum. Brain. 2006;129(Pt 2):290-2. doi: 10.1093/brain/ awh729.

15. De Zeeuw $\mathrm{Cl}$, Yeo $\mathrm{CH}$. Time and tide in cerebellar memory formation. Curr Opin Neurobiol. 2005;15(6):667-74. doi: 10.1016/j.conb.2005.10.008.

16. Timmann D, Watts S, Hore J. Failure of cerebellar patients to time finger opening precisely causes ball high-low inaccuracy in overarm throws. J Neurophysiol. 1999;82(1):103-14. doi: 10.1152/jn.1999.82.1.103. 